\title{
Development of a Diagnostic Questionnaire for Damp Phlegm Pattern and Blood Stasis Pattern in Coronary Heart Disease Patients (CHD-DPBSPQ)
}

\author{
Ge Fang $\mathbb{D}^{D}$, Ling-lin Zhang, Qi Ren, Xiao-wen Zhou, Bin Wang, Xuan Zhou $\mathbb{D}$, Xiao-qi Liu, \\ Dan-hong Peng, Xin-lin Chen $(\mathbb{D}$, and Xian-tao Li $\mathbb{B}$
}

School of Basic Medical Science, Guangzhou University of Chinese Medicine, Guangzhou, Guangdong Province 510006, China

Correspondence should be addressed to Xin-lin Chen; chenxlsums@126.com and Xian-tao Li; lxt150@126.com

Received 20 July 2019; Revised 9 October 2019; Accepted 25 October 2019; Published 26 November 2019

Academic Editor: I-Min Liu

Copyright $(2019$ Ge Fang et al. This is an open access article distributed under the Creative Commons Attribution License, which permits unrestricted use, distribution, and reproduction in any medium, provided the original work is properly cited.

\begin{abstract}
Background. The aim was to develop a diagnostic questionnaire for damp phlegm pattern and blood stasis pattern in coronary heart disease patients (CHD-DPBSPQ). Methods. The standard procedures of questionnaire development were carried out to develop and assess CHD-DPBSPQ. The patients were assessed using the CHD-DPBSPQ, CHD-DPPQ, and CHD-BSPQ. Four methods were used to select the items on the CHD-DPBSPQ in a pilot study based on data from a Guizhou tertiary grade A hospital. Cronbach's alpha and the split-half reliability, test-retest reliability, content validity, criterion validity, construct validity, and convergent validity were determined in a validation study using a nationwide sample. Results. After item selection, the CHDDPBSPQ contained 15 items in two domains: the phlegm domain (9 items) and the blood stasis domain (6 items). For the CHDDPBSPQ, the alpha coefficient was 0.88 , the split-half coefficient was 0.90 , and the intraclass correlation coefficient was 0.83 . The range of the item-level content validity index (I-CVI) was 0.71 to 1.0 and that of the scale-level content validity index/average (Scale-CVI/Ave) was 0.97. The domain scores on the CHD-DPBSPQ were in close relation to the scores on a questionnaire for damp phlegm pattern in coronary heart disease patients (CHD-DPPQ) and a questionnaire for blood stasis pattern in coronary heart disease patient (CHD-BSPQ) $(P<0.01)$. The root mean square error of approximation (RMSEA) was equal to 0.05 (90\% CI: $0.044,0.059)$. Convergent validity was demonstrated with a moderate correlation. Conclusion. The CHD-DPBSPQ is a reliable and valid instrument.
\end{abstract}

\section{Introduction}

Currently, coronary heart disease (CHD) is the most common type of cardiovascular disease (CVD) and is a major cause of death and disability among adults worldwide $[1,2]$. CHD causes approximately one-third of all deaths in people older than 35 years in Western countries [3]. CHD still affects more than 10 million people in China, and an estimated 7.4 million people suffer from CHD every year. In particular, approximately 3 million Chinese individuals die of CHD each year; the mortality rate is second only to that of cerebrovascular disease $[4,5]$. This may be related to increased serum cholesterol levels caused by smoking and dietary changes [6]. Under the influence of diabetes, hypertension, and hyperlipidaemia, the incidence of $\mathrm{CHD}$ increases annually.

Damp phlegm pattern and blood stasis pattern (DPBSP) is commonly found in CHD patients. Along with changes in lifestyle habits, DPBSP is becoming increasingly common [7-9]. Turbid phlegm and blood stasis are two important risk factors in the development of DPBSP. According to Chinese Medicine (CM), turbid phlegm is formed by body fluids, and its clinical manifestations include cough with sputum, chest tightness, dizziness, body fat accumulation, atherosclerosis, and hyperlipidaemia. Blood stasis is caused by illiquidity, and its clinical manifestations include tingling, localized pain, enclosed masses, dark purple tongue, and hemorheology [10]. Patients with CHD-DPBSP tend to have both 
phlegm and blood stasis pathological manifestations [11]. Bi et al. reported that DPBSP is found in $73.42 \%$ of $\mathrm{CHD}$ patients, and it is a primary syndrome in these patients [12].

TCM syndromes can be studied using measures such as scales. In recent years, some researchers have developed diagnostic questionnaires for CHD with stable angina (syndrome involving both phlegm and blood stasis) [13-15]. However, these questionnaires have some shortcomings. First, the process of developing these scales is not standardized. The method of determining construct validity is not suitable, as construct validity should be determined through confirmatory factor analysis (CFA) rather than exploratory factor analysis (EFA) [16]. Second, the items contained in these questionnaires are largely divergent; thus, the diagnosis of CHD is not systematic or consistent. For example, among the four diagnostic methods of TCM (inspection, listening and smelling, inquiry, and palpation), palpation was the main item in one diagnosis questionnaire [15], but we cannot find this aspect in others $[13,14]$. Some experts insist that tongue and pulse information are also very important for the diagnosis of DPBSP and can increase the diagnostic accuracy [17-21].

Therefore, we aimed to develop and assess a damp phlegm pattern and blood stasis pattern questionnaire for patients with coronary heart disease (CHD-DPBSPQ) based on a series of standard and systematic procedures of instrument development.

\section{Methods}

To develop the CHD-DPBSP diagnostic questionnaire, we used standard procedures for developing and validating the CHDDPBSPQ [22-29]. The procedures included the following steps: construct definition, item generation, a pilot study, and a validation study. Construct definition and item generation were used to define the structure and generate the initial items for item selection. As this methodology has been reported in other papers [30-33], it will not be reported in detail in this study. The pilot study was used to select the items for the CHDDPBSPQ, and the validation study was applied to assess the reliability and validity of the CHD-DPBSPQ.

2.1. Item Source. All the items on the CHD-DPBSPQ were drafted from systematic reviews, the Delphi method and analytic hierarchy process (AHP) [31-33]. Ultimately, 20 items were generated for CHD-DPBSPQ20 [34]. According to the theory of TCM, (1) chest distress, sleepiness, physical heaviness, obesity, sticky mouth, greasy tongue fur, slippery pulse, wiry pulse, abdominal fullness, anorexia, viscous stool, and taut and slippery pulse were used to assess the phlegm pattern. (2) Chest pain, cyanotic lips, dim complexion, dark purple tongue, petechiae or ecchymosis on the tongue, sublingual vein cyanosis, uneven pulse, and taut and uneven pulse were used to assess the blood stasis pattern in CHD patients.

\subsection{Pilot Study}

2.2.1. Samples. A pilot study was adopted to identify items for the CHD-DPBSPQ. Recruited from a Guizhou tertiary grade A hospital, all of the CHD patients with DPBSP provided informed consent prior to their participation. The eligibility criteria are as follows: (1) diagnosis of $\mathrm{CHD}$ according to the Chinese guidelines for diagnosed with $\mathrm{CHD}$ based on the Chinese guidelines for the diagnosis and treatment of patients with chronic stable angina published in 2007 [35]; (2) diagnosis of DPBSP by 2 experienced experts [36]; (3) $\geq 40$ years of age; and (4) provided informed consent for participation. Patients diagnosed with other syndromes or other diseases were excluded.

2.2.2. Analytical Methods. Four methods were employed to select the items for the CHD-DPBSPQ: (1) the coefficient of variation: if the standard deviation (SD) of every item was less than 0.9, the item was discarded; (2) EFA: if the correlation coefficient was less than 0.4 after factor rotation, the item was deleted; (3) alpha reduction: if Cronbach's alpha coefficient was apparently increased if one item was removed, that item was marked; and (4) correlation analysis: if the item had a proportion less than 0.4 , the item was abandoned [37-39]. If an item met three or more of the abovementioned criteria, it was removed.

\subsection{Validation Study}

2.3.1. National Sample. A multicentre dataset was used to assess the CHD-DPBSPQ. Patients from Guangdong Provincial Hospital of Traditional Chinese Medicine, Hunan University of Traditional Chinese Medicine, Hubei Provincial Hospital of Traditional Chinese Medicine, Affiliated Hospital of Shandong University of Traditional Chinese Medicine, Guizhou Provincial Hospital of Traditional Chinese Medicine, Second Affiliated Hospital of Wenzhou Medical University, Changzhou City Hospital of Traditional Chinese Medicine, and Tianjin University of Traditional Chinese Medicine were enrolled. All patients provided informed consent for participation. The inclusion criteria were as follows: (1) diagnosis of CHD on the basis of the Chinese guidelines for the diagnosis and treatment of patients with chronic stable angina published in 2007 [35]; (2) diagnosis of DPBSP or other syndromes by 2 experienced TCM doctors [36]; (3) $\geq 40$ years of age; and (4) provided informed consent for participation. The exclusion criteria were as follows: (1) unstable angina; (2) age $<40$ years or $>75$ years ; and (3) diagnosis of other diseases or other syndromes.

Two TCM doctors with over 20 years of experience in CVD performed the assessments, and the doctors explained the questionnaire to each patient. The questionnaire included a demographic portion, followed by the CHDDPBSPQ, CHD-DPPQ, and CHD-BSPQ. The demographic portion collected data on age, sex, ethnicity, marital status, occupation, and other diseases. The CHD-DPPQ and CHDBSPQ were used to evaluate the criterion validity. The CHDDPBSPQ was used for diagnosis by two experienced TCM doctors, and these two doctors could independently differentiate between the syndromes. If their diagnoses were inconsistent, diagnosis was made by a third doctor (associate professor or higher). Terwee et al. [40] believed that more 
than 50 samples should be assessed to determine test-retest reliability. At least 50 patients in ward settings were assessed by applying the CHD-DPBSPQ within 1-7 days, which is an applicable period to assess test-retest reliability.

2.3.2. Methods Used to Evaluate the Scale. SPSS version 22.0 and Amos software 22.0 were used for data analysis [41, 42]. This scale was evaluated using classical test theory (CTT), including reliability and validity measures.

Internal consistency reliability, split-half reliability, and test-retest reliability were all evaluated $[16,43]$. The internal consistency reliability was estimated using Cronbach's $\alpha$. After the arrangement of odd and even numbers, split-half reliability was calculated using Pearson's correlation coefficients. Test-retest reliability was assessed by adopting the intraclass correlation coefficient (ICC) and its 95\% confidence interval.

Validity was evaluated as content validity, criterion validity, construct validity, and convergent validity. The content validity of the questionnaire was assessed using the item-level content validity index (I-CVI) and the scale-level content validity index (S-CVI). When more than 6 experts assessed this questionnaire, the value of the I-CVI was not less than 0.78 [44-46]. We had 7 experts assess this questionnaire, and the value of the S-CVI/Ave exceeded 0.90, which indicated a high degree of content validity. The correlation coefficients between the CHD-DPPQ and the CHD-BSPQ were calculated to assess the criterion validity. CFA was used to evaluate whether the theoretical model was suitable for the data [28]. The goodness of fit index (GFI), adjusted goodness of fit index (AGFI), comparative fit index (CFI), and normed fit index (NFI) were greater than 0.9, indicating that the model was suitable. The root mean square error of approximation (RMSEA) was 0.05 , indicating that the fit was close to good [47]. The correlation coefficients of the subscales and items were equal to or greater than 0.4 , suggesting good convergence validity [48].

\section{Results}

3.1. Pilot Study. Ultimately, 103 CHD patients with DPBSP were involved in the pilot study. The age of the patients ranged from 46 years to 91 years (mean age: 68.8, Table 1); $60.2 \%$ of the sample was male, and $91.3 \%$ of the sample were retirees. Table 1 presents a brief demographic summary of the sample. The Cronbach's alpha coefficient of the CHDDPBSPQ20 was 0.835 . The results of item selection are shown in Table 2.

Of the 20 items, 5 items were removed from the original pool based on the selection criteria $(n \geq 3)$, namely, taut and slippery pulse, viscous stool, wiry pulse, uneven pulse, and taut and uneven pulse. Thus, CHD-DPBSPQ ultimately contained 15 items in 2 domains-the phlegm syndrome domain and the blood stasis syndrome domain. (1) The phlegm syndrome domain included chest distress, sleepiness, physical heaviness, obesity, sticky mouth, abdominal fullness, anorexia, greasy tongue fur, and slippery pulse. (2) The blood stasis syndrome domain included chest pain, cyanotic lips, dim complexion, dark purple tongue, petechiae or ecchymosis on the tongue, and sublingual vein cyanosis.

3.2. Validation Study. To further improve the demographic composition of the sample, data were collected from the clinical population of 8 hospitals in China. The demographic characteristics of the 729 total participants are shown in Table 1. Statistically, the mean age was $67.5 \pm 10.6$ years (range, 32.0-91.0 years), and a total of 460 male and 269 female patients were included. Among these patients, 99.3\% were of Han ethnicity, $67.5 \%$ were retirees, $99.5 \%$ were married, and $88.0 \%$ had another disease. The sample $(N=729)$ was measured for the first time, with 81 of the patients measured a second time.

Table 2 shows the item distributions, which were assessed using means, standard deviation (SD), and missing data. In total, 729 patients were enrolled and completed the questionnaire. All the item scores were between 0 and 4 (Table 3). Chest distress had the highest score (1.94), while sleepiness had the lowest $(0.45)$. Chest pain, sticky mouth, and cyanotic lips had missing values.

3.2.1. Reliability. The CHD-DPBSP had high internal consistency and retest reliability. The mean score on the CHDDPBSPQ was 17.4, the mean score on the phlegm domain was 9.7, and the mean score on the blood stasis domain was 7.7 (Table 4). (1) The Cronbach's alpha values for both domains were greater than 0.75 (Table 4). (2) The split-half coefficients of the domains were greater than 0.80 (Table 4). (3) The interitem correlation coefficient (ICC) of the domains were $0.78,0.83$, and 0.83 , which were more than 0.8 , and the retest reliability coefficient was high (Table 4 ).

3.2.2. Validity. The CHD-DPBSPQ had good validity. (1) For content validity, the range of the I-CVI was 0.71 to 1.0 , and the S-CVI/Ave was 0.97 (Table 5). (2) The criterion validity was checked by comparing the scale with the CHDDPPQ and the CHD-BSPQ. The correlation coefficient between the phlegm domain and the CHD-DPPQ was 0.76, and the correlation coefficient between the blood stasis domain and the CHD-BSPQ was $0.96(P<0.01)$. (3) The model fit for the scale was tested using CFA (Figure 1). The GFI, AGFI, NFI, IFI, TLI, and CFI were all greater than 0.90, and the RMSEA was 0.05 (90\% CI: 0.044, 0.059) (Table 6). All these indexes indicated that the model fit was good.(4) Convergent validity was demonstrated by a moderate correlation (0.423-0.796), as shown in Table 7.

\section{Discussion}

At present, the specific scales used in patients with $\mathrm{CHD}$ abroad are the Seattle angina scale (SAQ) [49] and the cardiovascular limitations and symptoms profile (CLASP) [50]. Nevertheless, owing to cultural differences, we researched and developed the CHD-DPBSPQ to diagnose DPBSP in Chinese CHD patients. 
TABLE 1: Demographic characteristics of the patients with CHD-DPBSP.

\begin{tabular}{|c|c|c|c|}
\hline \multirow{2}{*}{ Characteristic } & \multirow{2}{*}{$\begin{array}{c}\text { Pilot study } \\
\text { CHD-DPBSP }(\%, n=103)\end{array}$} & \multicolumn{2}{|c|}{ Validation study } \\
\hline & & CHD-DPBSP $(\%, n=729)$ & CHD-DPBSP $(\%, n=81)^{*}$ \\
\hline Age, mean \pm SD (range) & $68.8 \pm 9.2(46,91)$ & $67.5 \pm 10.6(32.0,91.0)$ & $66.9 \pm 12.2(38.0,90.0)$ \\
\hline \multicolumn{4}{|l|}{ Sex } \\
\hline Male & $62(60.2)$ & $460(63.1)$ & $48(59.3)$ \\
\hline Female & $41(39.8)$ & $269(36.9)$ & $33(40.7)$ \\
\hline \multicolumn{4}{|l|}{ Ethnicity } \\
\hline Han & $101(98.1)$ & $724(99.3)$ & $81(100.0)$ \\
\hline Others & $2(1.9)$ & $5(0.7)$ & $0(0)$ \\
\hline \multicolumn{4}{|l|}{ Marital status } \\
\hline Married & $103(100)$ & $725(99.5)$ & $100(100.0)$ \\
\hline Unmarried & $0(0)$ & $4(0.5)$ & $0(0)$ \\
\hline \multicolumn{4}{|l|}{ Occupation } \\
\hline Worker & $0(0)$ & $66(9.1)$ & $9(11.1)$ \\
\hline Farmer & $3(2.9)$ & $73(10.0)$ & $7(8.6)$ \\
\hline Specialist & $3(2.9)$ & $26(3.6)$ & $2(2.5)$ \\
\hline Staff & $3(3.9)$ & $67(9.2)$ & $11(13.6 .0)$ \\
\hline Retiree & $94(91.3)$ & $497(67.5)$ & $52(64.2)$ \\
\hline \multicolumn{4}{|l|}{ Other disease } \\
\hline Yes & $85(82.5)$ & $641(88.0)$ & $71(87.7)$ \\
\hline No & $18(17.5)$ & $88(12.0)$ & $10(12.3)$ \\
\hline
\end{tabular}

CHD-DPBSP: damp phlegm pattern and blood stasis pattern for coronary heart disease. ${ }^{*}$ These patients were used for the retest.

TABLE 2: The results of item selection (pilot study).

\begin{tabular}{|c|c|c|c|c|c|}
\hline Items & $\mathrm{CV}$ & EFA & $\alpha$ value & $r$ & Deleted item \\
\hline Chest distress & 0.531 & 0.605 & 0.670 & 0.642 & \\
\hline Chest pain & 1.115 & 0.718 & 0.752 & 0.757 & \\
\hline Sleepiness & 1.100 & 0.286 & 0.689 & 0.465 & \\
\hline Physical heaviness & 0.984 & 0.613 & 0.658 & 0.625 & \\
\hline Obesity & 1.237 & 0.506 & 0.669 & 0.587 & \\
\hline Sticky mouth & 1.171 & 0.752 & 0.665 & 0.594 & \\
\hline Cyanotic lips & 0.725 & 0.740 & 0.747 & 0.769 & \\
\hline Dim complexion & 0.999 & 0.849 & 0.712 & 0.881 & \\
\hline Abdominal fullness & 1.066 & 0.782 & 0.632 & 0.748 & \\
\hline Anorexia & 1.075 & 0.717 & 0.645 & 0.686 & \\
\hline Viscous stool & 0.651 & -0.180 & 0.710 & 0.183 & $x$ \\
\hline Dark purple tongue & 0.666 & 0.773 & 0.747 & 0.785 & \\
\hline Petechiae or ecchymosis on the tongue & 1.290 & 0.859 & 0.730 & 0.849 & \\
\hline Sublingual vein cyanosis & 0.815 & 0.782 & 0.729 & 0.843 & \\
\hline Greasy tongue fur & 0.847 & 0.779 & 0.644 & 0.730 & \\
\hline Slippery pulse & 0.976 & 0.729 & 0.642 & 0.713 & \\
\hline Wiry pulse & 1.031 & -0.485 & 0.749 & 0.026 & $x$ \\
\hline Uneven pulse & 0.429 & -0.328 & 0.833 & -0.147 & $x$ \\
\hline Taut and slippery pulse & 1.240 & -0.503 & 0.771 & -0.003 & $x$ \\
\hline Taut and uneven pulse & 0.589 & -0.350 & 0.846 & -0.113 & $x$ \\
\hline
\end{tabular}

$\mathrm{CV}$ : coefficient of variation; EFA: exploratory factor analysis; $\alpha$ value: alpha reduction; $r$ : correlation analysis. If the item met three or more of the abovementioned criteria, it was deleted.

Previous instruments for the assessment of CHD-DPBSP have not been widely adopted; our group formulated and validated TCM outcomes on the basis of standard development and validation procedures [22, 28, 40]. The US FDA and the WHOQOL group proposed the establishment of a conceptual framework for questionnaires [23, 26]. According to TCM theories, the framework for DPBSP should be classified into damp phlegm and blood stasis patterns $[10,11]$. The diagnosis of DPBSP requires the simultaneous diagnosis of sputum and blood stasis
[11, 20, 51, 52]. However, these questionnaires [13-15] all contain different frameworks. For example, one questionnaire involved the division of dimensions into phlegm and blood stasis [14], but the other two did not [13, 15]. Moreover, some important validity coefficients, such as convergent validity, were not evaluated. The method of determining content validity was not suitable, as content validity should be determined according to the CVI [45], but none of the three questionnaires used the CVI [13-15]. The method of construct validity was also not suitable, as 
TABLE 3: Lacking data, mean, and SD for each item $(n=729)$.

\begin{tabular}{|c|c|c|c|c|c|c|c|}
\hline Item & Score 0 & Score 1 & Score 2 & Score 3 & Lacking & Mean & $\mathrm{SD}$ \\
\hline Chest distress & 99 & 116 & 245 & 269 & 0 & 1.94 & 1.03 \\
\hline Chest pain & 247 & 141 & 190 & 150 & 1 & 1.33 & 1.15 \\
\hline Sleepiness & 527 & 102 & 71 & 29 & 0 & 0.45 & 0.83 \\
\hline Physical heaviness & 316 & 127 & 236 & 50 & 0 & 1.03 & 1.02 \\
\hline Obesity & 325 & 142 & 206 & 56 & 0 & 0.99 & 1.02 \\
\hline Sticky mouth & 348 & 164 & 137 & 79 & 1 & 0.93 & 1.05 \\
\hline Cyanotic lips & 269 & 189 & 167 & 103 & 1 & 1.14 & 1.07 \\
\hline Dim complexion & 275 & 179 & 166 & 109 & 0 & 1.15 & 1.09 \\
\hline Abdominal fullness & 414 & 115 & 151 & 49 & 0 & 0.77 & 1 \\
\hline Anorexia & 429 & 132 & 127 & 41 & 0 & 0.7 & 0.95 \\
\hline Dark purple tongue & 196 & 125 & 264 & 144 & 0 & 1.49 & 1.09 \\
\hline Petechiae or ecchymosis on the tongue & 297 & 130 & 167 & 135 & 0 & 1.19 & 1.16 \\
\hline Sublingual vein cyanosis & 191 & 188 & 214 & 136 & 0 & 1.4 & 1.07 \\
\hline Greasy tongue fur & 120 & 158 & 302 & 149 & 0 & 1.66 & 0.98 \\
\hline Slippery pulse & 308 & 93 & 191 & 137 & 0 & 1.22 & 1.18 \\
\hline
\end{tabular}

SD: standard deviation.

TABLE 4: Descriptive statistical data and reliability of the CHD-DPBSPQ.

\begin{tabular}{lccccrr}
\hline & No. of items & Mean \pm SD & Range of score & Cronbach's alpha & Split-half coefficient & ICC (95\% CI) \\
\hline Phlegm domain & 9 & $9.7 \pm 5.4$ & $(0.0,27.0)$ & 0.77 & 0.81 & $0.78(0.66,0.86)$ \\
Blood stasis domain & 6 & $7.7 \pm 4.7$ & $(0.0,18.0)$ & 0.81 & 0.81 & $0.83(0.74,0.89)$ \\
CHD-DPBSPQ & 15 & $17.4 \pm 9.5$ & $(0.0,45.0)$ & 0.88 & 0.90 & $0.83(0.74,0.89)$ \\
\hline
\end{tabular}

ICC: intraclass correlation coefficient.

TABLE 5: The values of I-CVI for each item and S-CVI/Ave for the questionnaire.

\begin{tabular}{lcc}
\hline Item & I-CVI & No. of respondents \\
\hline Chest distress & 1.00 & 7 \\
Chest pain & 1.00 & 7 \\
Sleepiness & 1.00 & 7 \\
Physical heaviness & 1.00 & 7 \\
Obesity & 1.00 & 7 \\
Sticky mouth & 0.71 & 7 \\
Cyanotic lips & 1.00 & 7 \\
Dim complexion & 0.86 & 7 \\
Abdominal fullness & 1.00 & 7 \\
Anorexia & 1.00 & 7 \\
Dark purple tongue & 1.00 & 7 \\
Petechiae or ecchymosis on the tongue & 1.00 & 7 \\
Sublingual vein cyanosis & 1.00 & 7 \\
Greasy tongue fur & 1.00 & 7 \\
Slippery pulse & 1.00 & 7 \\
S-CVI/Ave & $0.97^{*}$ & \\
\hline
\end{tabular}

I-CVI: item-level content validity index. *: scale-level content validity index, scale-level CVI; average (scale-CVI/Ave).

construct validity should be determined via CFA rather than EFA [16].

The 15 items on the CHD-DPBSPQ were empirically selected by four methods using a sample population from a Guizhou tertiary grade A hospital. The CHD-DPBSPQ was verified with national data, showing that it is a reliable and valid tool for research and clinical trials. (1) The internal consistency of the domains was expressed by Cronbach's $\alpha$ coefficients $(0.77-0.88)$ and split-half reliability coefficients (0.81-0.90). Terwee et al. considered that the Cronbach's $\alpha$ showed a good internal consistency range, from 0.70 to 0.95 [40], and the CHD-DPBSPQ had good internal consistency. Moreover, eighty-one patients completed the CHDDPBSPQ a second time, and the ICC was between 0.78 and 0.83 , indicating good reproducibility [40].

(2) The content validity of the questionnaire was evaluated by seven experts. A standard value of I-CVI greater than 0.78 was recommended by Lynn [53]; the score for sticky mouth was 0.71 , but the Kappa-like index $(K)$ was 0.65 (>0.60), which showed good validity [54]. The S-CVI for the 


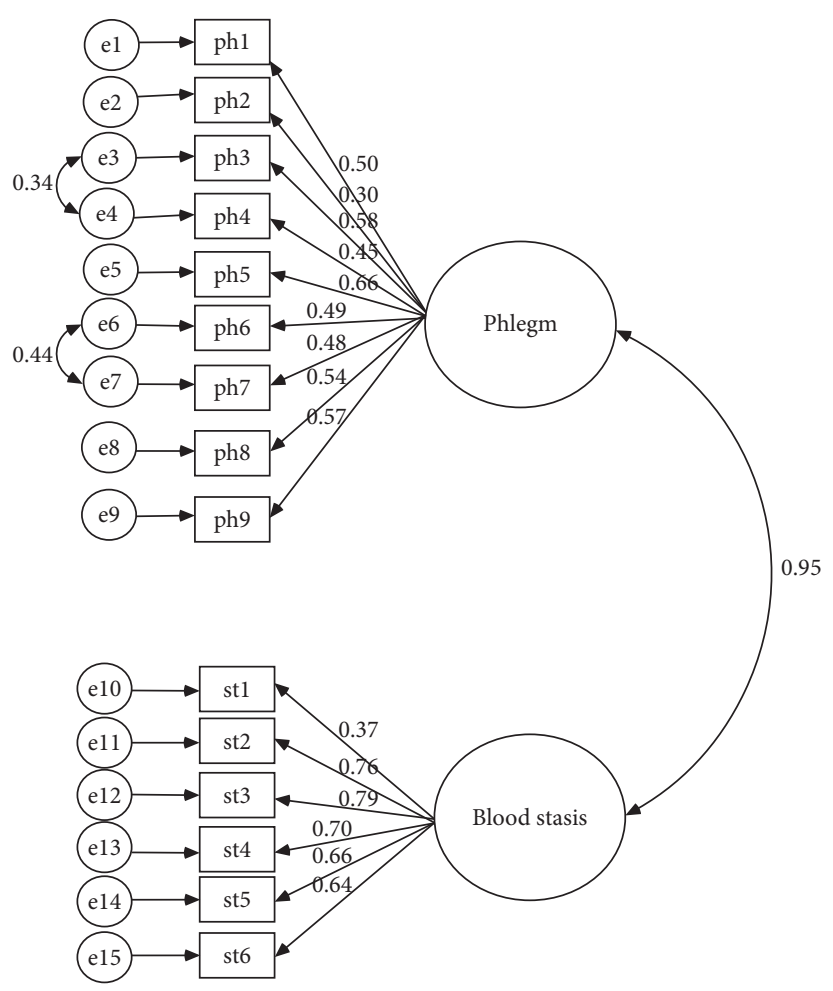

FIGURE 1: Standardized regression weight in the questionnaire confirmatory factor analysis model. ph1: chest distress, ph2: sleepiness, ph3: physical heaviness, ph4: obesity, ph5: sticky mouth, ph6: abdominal fullness, ph7: anorexia, ph8: greasy fur, and ph9: slippery pulse; st1: chest pain, st2: cyanotic lips, st3: dim complexion, st4: dark purple tongue, st5: petechiae or ecchymosis on the tongue, and st6: sublingual vein cyanosis.

TABLE 6: Model fit test results for the CFA model.

\begin{tabular}{lcccccccc}
\hline Models & $\chi^{2} / \mathrm{d} f$ & RMSEA & GFI & AGFI & NFI & IFI & TLI & CFI \\
\hline $\begin{array}{l}\text { 2-factor } \\
\text { model }\end{array}$ & 2.90 & 0.05 & 0.96 & 0.94 & 0.93 & 0.95 & 0.94 & 0.95 \\
\hline
\end{tabular}

CFA: confirmatory factor analysis, RMSEA: root mean square error of approximation, GFI: goodness of fit index, AGFI: adjusted goodness of $\mathrm{ft}$ index, NFI: normed fit index, IFI: increment fitting index, TLI: TurkerLewis index, CFI: comparative fit index.

scale was 0.97 , which meets the requirements of the standard value (0.90) recommended by Polit and Beck [55]. The correlation coefficient between the phlegm domain and the CHD-DPPQ was 0.76 , and the correlation coefficient between the blood stasis domain and the CHD-BSPQ was 0.96. Their values were greater than 0.7 , which indicated positive criterion validity [40]. The RMSEA was equal to $0.05(90 \%$ CI: $0.044,0.059)$, and the NFI, NNFI, and CFI were greater than 0.90. These results indicated that the scale had good construct validity [47]. The correlations of the CHDDPBSPQ between the phlegm domain and our hypothesized components (chest distress, sleepiness, physical heaviness, obesity, sticky mouth, abdominal fullness, anorexia, greasy tongue fur, and slippery pulse) were greater than 0.4 , as were the correlations of the CHD-DPBSPQ between the blood stasis domain and our hypothesized components (chest pain, cyanotic lips, dim complexion, dark purple tongue, petechiae or ecchymosis on the tongue, and sublingual vein cyanosis), indicating moderate convergence validity [48]. In short, these findings are encouraging and support the structural integrity of the CHD-DPBSPQ.

The purpose of our study is to develop and validate the CHD-DPBSPQ, a questionnaire intended for use in the clinical practice of TCM. However, the questionnaire is not applicable for diagnosing CHD and all TCM syndromes of $\mathrm{CHD}$. Thus, the diagnosis of CHD was first confirmed in patients recruited for our study according to the Chinese guidelines for the diagnosis of $\mathrm{CHD}$, which were based on the Chinese guidelines for the diagnosis and treatment of patients with chronic stable angina published in 2007 [35]. The CHD-DPBSPQ was developed on the basis of the theory of TCM syndromes. Meanwhile, structural equation modelling is a valid method for testing TCM syndromes, as described in other studies [23, 29]. Up to now, other researchers have formulated and exerted similar methods to explore TCM syndromes $[16,24]$. For example, Chen et al. developed the LIDHS questionnaire and evaluated its reliability and validity as a "TCM Syndrome Questionnaire of Ulcerative Colitis" [16]. The CHD-DPBSPQ, as an effective and reliable pattern of TCM questionnaire, can also be used to diagnose CHD-DPBSP in patients.

There were a few limitations. (1) The patients were retested again 1-7 days after the first investigation. Thus, test-retest reliability could have been overestimated due to the short time interval. (2) No pathological data were provided for the diagnosis of CHD-DPBSP. (3) The pilot study enrolled patients from a Guizhou tertiary grade A 
TABLE 7: Correlation coefficients between each item and different domains.

\begin{tabular}{lcc}
\hline & Phlegm domain & Blood stasis domain \\
\hline Phlegm & & $0.571^{* *}$ \\
Chest distress & $0.423^{* *}$ & 0.435 \\
Sleepiness & $0.687^{* *}$ & 0.244 \\
Physical heaviness & $0.569^{* *}$ & 0.509 \\
Obesity & $0.695^{* *}$ & 0.411 \\
Sticky mouth & $0.625^{* *}$ & 0.569 \\
Abdominal fullness & $0.586^{* *}$ & 0.423 \\
Anorexia & $0.584^{* *}$ & 0.432 \\
Greasy tongue fur & $0.608^{* *}$ & 0.472 \\
Slippery pulse & & 0.507 \\
\hline Blood stasis & 0.345 & $0.538^{* *}$ \\
Chest pain & 0.580 & $0.786^{* *}$ \\
Cyanotic lips & 0.642 & $0.796^{* *}$ \\
Dim complexion & 0.564 & $0.748^{* *}$ \\
Dark purple tongue & 0.559 & $0.734^{* *}$ \\
Petechiae or ecchymosis on the tongue & 0.556 & $0.706^{* *}$ \\
Sublingual vein cyanosis & & \\
\hline
\end{tabular}

${ }^{* *} P$ value $<0.01$.

hospital. The CHD-DPBSPQ should be further assessed throughout every province in the country. (4) The gold standard adopted was the CHD-DPPQ and CHD-BSPQ developed in China, which do not include the internationally recognized scale for $\mathrm{CHD}$.

This questionnaire also has some strengths. First, compared with one published scale [13], our questionnaire was more convincing in terms of verification. Second, the development of a diagnostic CHD-DPBSPQ is rare. Third, $100 \%$ of the patients completed the questionnaire. Fourth, the content of our scale was easy to understand, and the average completion time for the questionnaire was 5.4 minutes. Finally, it was confirmed that the results were generalizable by using a multicentre clinical investigation.

\section{Conclusions}

A DPBSP questionnaire was developed for patients with $\mathrm{CHD}$. The 20 items were successfully reduced to 15 items during the selection process, a quantitative stage of questionnaire development. The CHD-DPBSPQ showed good reliability and validity and is feasible for diagnosing DPBSP in CHD patients. We recommend the application of the CHD-DPBSPQ for diagnosing DPBSP in Chinese CHD patients.

\section{Abbreviations}

CHD- Questionnaire of damp phlegm pattern and DPBSPQ: blood stasis pattern for coronary heart disease CVI: $\quad$ Content validity index

I-CVI: Item-level content validity index

S-CVI: $\quad$ Scale-level content validity index

CHD- Questionnaire of damp phlegm pattern for

DPPQ: $\quad$ coronary heart disease

CHD- Questionnaire of blood stasis syndrome for

BSPQ: $\quad$ coronary heart disease

CM: $\quad$ Chinese medicine
CFA: Confirmatory factor analysis

QOL: Quality of life

SD: $\quad$ Standard deviation

CTT: Classical test theory

ICC: Intraclass correlation coefficient

RMSEA: Root mean square error of approximation

DPBSPQ: Damp phlegm pattern and blood stasis pattern

GFI: $\quad$ Goodness of fit index

AGFI: $\quad$ Adjusted goodness of fit index

CFI: $\quad$ Comparative fit index

NFI: $\quad$ Normed fit index

RMSEA: Root mean square error of approximation

SAQ: $\quad$ Seattle angina scale

CLASP: Cardiovascular limitations and symptoms profile.

\section{Data Availability}

The data used to support the findings of this study are available from the corresponding author upon request.

\section{Ethical Approval}

The Research Ethics Committee of Tianjin University of Traditional Chinese Medicine provided approval for this study (no. TJUTCMEC2015000).

\section{Conflicts of Interest}

The authors declare that there are no conflicts of interest regarding the publication of this paper.

\section{Authors' Contributions}

Ge Fang participated in the study design, analysed the data, and wrote and modifed the manuscript. Ling-lin Zhang participated in the study design and drafted the manuscript. Qi Ren and Xiao-wen Zhou helped to modify the 
manuscript. Bin Wang and Xuan Zhou participated in the study design and data collection. Xiao-qi Liu and Dan-hong Peng participated in the data collection. Xin-lin Chen greatly contributed to the data analysis and manuscript review. Xian-tao Li greatly contributed to the conception of the study and study design. All authors read and approved the final manuscript.

\section{Acknowledgments}

This study was supported by the National Basic Research Programme of China (973 Programme) (2014CB542901). The authors would like to thank the following teams for their valuable contributions: the Yang Xiaobo Research Team at Guangdong Provincial Hospital of Traditional Chinese Medicine; the Hu Zhixi Research Team at Hunan University of Traditional Chinese Medicine; the Wu Bin Research Team at Hubei Provincial Hospital of Traditional Chinese Medicine; Dai Guohua Research Team of the Affiliated Hospital of Shandong University of Chinese Medicine; the Sun Gang Research Team at Guizhou Provincial Hospital of Traditional Chinese Medicine; the Zheng Guoqing Research Team at the Second Affiliated Hospital of Wenzhou Medical University; the Qian Weidong Research Team at Changzhou City Hospital of Traditional Chinese Medicine; and the Yu Chunquan Research Team at Tianjin University of Traditional Chinese Medicine.

\section{References}

[1] E. J. Benjamin, P. Muntner, A. Alonso et al., "Heart disease and stroke statistics-2019 update: a report from the American Heart Association," Circulation, vol. 139, no. 10, pp. e56-e528, 2019.

[2] J. A. Leigh, M. Alvarez, and C. J. Rodriguez, "Ethnic minorities and coronary heart disease: an update and future directions," Current Atherosclerosis Reports, vol. 18, no. 2, p. 9 , 2016.

[3] M. Nichols, N. Townsend, P. Scarborough, and M. Rayner, "Cardiovascular disease in Europe 2014: epidemiological update," European Heart Journal, vol. 35, no. 42, pp. 29502959, 2014.

[4] S. S. Hu, L. Z. Kong, R. L. Gao et al., "Outline of the report on cardiovascular disease in China, 2010," Biomedical and Environmental Sciences, vol. 25, no. 3, pp. 251-256, 2012.

[5] W. W. Chen, R. L. Gao, L. S. Liu et al., "China cardiovascular diseases report 2015: a summary," Journal of Geriatric Cardiology, vol. 14, no. 1, pp. 1-10, 2017.

[6] J. E. Dalen, J. S. Alpert, R. J. Goldberg, and R. S. Weinstein, "The epidemic of the 20th century: coronary heart disease," The American Journal of Medicine, vol. 127, no. 9, pp. 807-812, 2014.

[7] R. Yuan, J. Wang, and L. L. Guo, "Effect of traditional Chinese medicine on coronary heart disease with phlegm and blood stasis syndrome," China Journal of Chinese Materia Medica, vol. 41, no. 1, pp. 35-37, 2016.

[8] X. Y. Lu and H. X. Cao, "Discussion on the treatment of coronary heart disease from "phlegm and blood stasis," Journal of Traditional Chinese Medicine, vol. 51, no. 2, pp. 101-103, 2010.

[9] G. H. Li, H. Y. Jiang, Y. N. Xie et al., “Analysis of 84697 TCM syndromes of coronary heart disease and the use of Western medicine based on big data," China Journal of Chinese Materia Medica, vol. 39, no. 18, p. 3462, 2014.

[10] W. F. Zhu and Q. H. He, Modern Chinese Medicine Clinical Diagnostics, People's Health Publishing House, Beijing, China, 2003.

[11] J. Q. Hu, C. C. Wang, F. Duan et al., "Study on macrocosmic diagnostic criteria for coronary heart disease with intermingled phlegm-blood stasis syndrome," Chinese Journal of Integrated Traditional \& Western Medicine, vol. 36, no. 10, pp. 1164-1168, 2016.

[12] Y. F. Bi, X. L. Wang, J. Y. Mao, and B. L. Zhang, "Diagnosis of traditional Chinese medicine syndrome of coronary heart disease with angina pectoris based on clinical epidemiological survey," Journal of Traditional Chinese Medicine, vol. 59, no. 22, pp. 1977-1980, 2018.

[13] L. X. Liu, "The development and evaluation of the scale of stable angina (the syndrome of phlegm and blood stasis in TCM)," Master, Liaoning University of Traditional Chinese Medicine, Shenyang, China, 2011.

[14] M. J. Lv, "Development and evaluation of syndrome differentiation scale for coronary heart disease stable angina pectoris (phlegm and blood stasis syndrome)," Master, Liaoning University of Traditional Chinese Medicine, Shenyang, China, 2014.

[15] H. L. Wang, "Development and preliminary evaluation of syndrome evaluation scores for coronary heart disease stable angina pectoris (syndrome of intermingled phlegm and blood stasis)," Master, Liaoning Journal of traditional Chinese Medicine, Shenyang, China, 2014.

[16] X. L. Chen, Y. Wen, Z. C. Wu et al., "Development of a traditional Chinese medicine syndrome-specific scale for ulcerative colitis: the large intestine dampness-heat syndrome questionnaire," Evidence-Based Complementary and Alternative Medicine, vol. 2018, Article ID 4039019, 10 pages, 2018.

[17] S. G. Shen, "New ideas for the diagnosis and treatment of coronary heart disease," Journal of Emergency in Traditional Chinese Medicine, vol. 8, no. 2, pp. 51-53, 1999.

[18] S. G. Shen and X. J. Han, "Name correction and standardization of TCM heart disease," Chinese Journal of Basic Medicine in Traditional Chinese Medicine, vol. 13, no. 7, pp. 485-487, 2007.

[19] X. J. Han and Y. S. Zhang, "Origin and innovation of syndrome of phlegm and blood stasis in coronary heart disease," China Journal of Traditional Chinese Medicine and Pharmacy, vol. 19, no. 10, pp. 623-625, 2004.

[20] Z. H. Zhao, TCM Therapeutics of Coronary Heart Disease, China Medical Science Press, Beijing, China, 2013.

[21] Y. Wang, J. Xu, R. Guo et al., "Therapeutic effect in patients with coronary heart disease based on information analysis from traditional Chinese medicine four diagnostic methods," Journal of Traditional Chinese Medicine, vol. 34, no. 1, pp. 34-41, 2014.

[22] K.-f. Leung, F.-b. Liu, L. Zhao, J.-q. Fang, K. Chan, and L.-z. Lin, "Development and validation of the Chinese quality of life instrument," Health and Quality of Life Outcomes, vol. 3, no. 1, p. 26, 2005.

[23] N. L. Zhang, S. Yuan, T. Chen, and Y. Wang, "Latent tree models and diagnosis in traditional Chinese medicine," Artificial Intelligence in Medicine, vol. 42, no. 3, pp. 229-245, 2008.

[24] Y.-X. Yan, Y.-Q. Liu, M. Li et al., "Development and evaluation of a questionnaire for measuring suboptimal health status in urban Chinese," Journal of Epidemiology, vol. 19, no. 6, pp. 333-341, 2009. 
[25] F.-b. Liu, X.-l. Chen, L. Guo, and X.-b. Liu, "Evaluation of a scale of patient-reported outcomes for the assessment of myasthenia gravis patients in China," Chinese Journal of Integrative Medicine, vol. 18, no. 10, pp. 737-745, 2012.

[26] T. Tomura, K. Yoshimasu, J. Fukumoto et al., "Validity of a diagnostic scale for acupuncture: application of the item response theory to the five viscera score," Evidence-Based Complementary and Alternative Medicine, vol. 2013, Article ID 928089, 11 pages, 2013.

[27] Q. Guo and Q. Chen, "Standardization of syndrome differentiation defined by traditional Chinese medicine in operative breast cancer: a modified Delphi study," Scientific World Journal, vol. 2015, Article ID 820436, 5 pages, 2015.

[28] Y. Su, C. W. Mo, W. Q. Cheng et al., "Development and validation of quality of life scale of nasopharyngeal carcinoma patients: the QOL-NPC (version 2)," Health and Quality of Life Outcomes, vol. 14, no. 1, p. 76, 2016.

[29] R. Q. Chen, C. M. Wong, and T. H. Lam, "Construction of a traditional Chinese medicine syndrome-specific outcome measure: the kidney deficiency syndrome questionnaire (KDSQ)," BMC Complementary and Alternative Medicine, vol. 12, no. 1, p. 73, 2012.

[30] X. T. Li, "Understanding of clinical syndrome differentiation of phlegm and blood stasis syndrome (chest pain)," Liaoning Journal of Traditional Chinese Medicine, vol. 43, no. 1, pp. 10-12, 2016.

[31] Y. P. Wang, F. F. Tang, X. Zhou, B. Wang, and G. Fang, "Literature system evaluation of phlegm and blood stasis syndrome of coronary heart disease," Tianjin Journal of Traditional Chinese Medicine, vol. 36, no. 4, pp. 322-327, 2019.

[32] R. Xie, X. F. Lian, X. Zhou et al., "Assessing for syndrome differentiation basis of intermingled phlegm-blood stasis syndrome in coronary heart disease based on Delphi method," Tianjin Journal of Traditional Chinese Medicine, vol. 36, no. 3, pp. 214-217, 2019.

[33] R. Xie, X. F. Lian, X. Zhou et al., "Preliminary assessment based on analytic hierarchy process on the importance of syndrome differentiation basis of intermingled phlegm-blood stasis syndrome in coronary heart disease," Tianjin Journal of Traditional Chinese Medicine, vol. 36, no. 1, pp. 23-26, 2019.

[34] X. Q. Liu, D. H. Peng, Y. P. Wang et al., "Diagnostic accuracy of Chinese medicine diagnosis scale of phlegm and blood stasis syndrome in coronary heart disease: a study protocol," Chinese Journal of Integrative Medicine, vol. 25, no. 7, pp. 515-520, 2019.

[35] Chinese Society of Cardiology (Chinese Medical Association), "Guideline for diagnosis and treatment of patients with chronic stable angina," Chinese Journal of Cardiology, vol. 35, no. 3, pp. 195-206, 2007.

[36] Z. Wang, X.-H. Zhou, and M. Wang, "Evaluation of diagnostic accuracy in detecting ordered symptom statuses without a gold standard," Biostatistics, vol. 12, no. 3, pp. 567-581, 2011.

[37] X. C. Guo, J. P. Zhang, Y. P. Zhu et al., "Item selection analysis based on quality of life scale in patients with viral myocarditis," Acta Academiae Medicinae Sinicae, vol. 34, no. 2, pp. 116-125, 2012.

[38] A. Tatar, G. Saltukoglu, S. Alioglu, S. Cimen, H. Guven, and C. E. Ay, "Measuring alexithymia via trait approach-I: a alexithymia scale item selection and formation of factor structure," Noro Psikiyatri Arsivi, vol. 54, no. 3, pp. 216-224, 2017.
[39] M. F. Gu, Y. Z. Du, X. L. Chen et al., "Item selection in the development of quality of life scale for nasopharyngeal carcinoma patients," Chinese Journal of Cancer, vol. 28, no. 1, pp. 103-106, 2009.

[40] C. B. Terwee, S. D. M. Bot, M. R. de Boer et al., "Quality criteria were proposed for measurement properties of health status questionnaires," Journal of Clinical Epidemiology, vol. 60, no. 1, pp. 34-42, 2007.

[41] A. Le, L. Zhang, W. Liu, X. Li, J. Ren, and A. Ning, "A case control study on the structural equation model of the mechanism of coagulation and fibrinolysis imbalance in chronic schistosomiasis," Medicine, vol. 96, no. 7, Article ID e6116, 2017.

[42] M. I. Mahmood, S. A. Shah, N. Ahmad, and N. M. Rosli, "Cancer screening perception scale: development and construct validation," Journal of Cancer Education, vol. 33, no. 2, pp. 269-277, 2018.

[43] Z. C. Tezcaner and S. Aksoy, "Reliability and validity of the Turkish version of the voice-related quality of life measure," Journal of Voice, vol. 31, no. 2, pp. 262 e267-262 e211, 2017.

[44] M. S. Choi and H. Shin, "Reliability and validity of the menopausal symptom scale," Women \& Health, vol. 55, no. 1, pp. 58-76, 2015.

[45] R. J. Hawkins, B. Swanson, M. J. Kremer, and L. Fogg, "Content validity testing of questions for a patient satisfaction with general anesthesia care instrument," Journal of PeriAnesthesia Nursing, vol. 29, no. 1, pp. 28-35, 2014.

[46] R. Farzanegan, B. Farzanegan, M. Alehashem et al., "Item selection and content validity of the risk factors of post-intubation tracheal stenosis observation questionnaire for ICUadmitted patients," Tanaffos, vol. 16, no. 1, pp. 22-33, 2017.

[47] M. W. Browne and R. Cudeck, "Alternative ways of assessing model fit," Sociological Methods \& Research, vol. 21, no. 2, pp. 230-258, 2016.

[48] K. N. Zhou, M. Zhang, Q. Wu et al., "Reliability, validity and sensitivity of the Chinese (simple) short form 36 health survey version 2 (SF-36v2) in patients with chronic hepatitis B," Journal of Viral Hepatitis, vol. 20, no. 4, pp. e47-e55, 2013.

[49] J. A. Spertus, J. A. Winder, T. A. Dewhurst et al., "Development and evaluation of the Seattle Angina questionnaire: a new functional status measure for coronary artery disease," Journal of the American College of Cardiology, vol. 25, no. 2, pp. 333-341, 1995.

[50] R. J. Lewin, D. R. Thompson, C. R. Martin et al., "Validation of the cardiovascular limitations and symptoms profile (CLASP) in chronic stable angina," Journal of Cardiopulmonary Rehabilitation, vol. 22, no. 3, pp. 184-191, 2002.

[51] H. L. Wu, Heart Spleen Theory and Cardiovascular Diseases, People's Health Publishing House, Beijing, China, 2005.

[52] W. Q. Zhang, Coronary Heart Disease, People's Health Publishing House, Beijing, China, 1982.

[53] M. R. Lynn, "Determination and quantification of content validity,” Nursing Research, vol. 35, no. 6, pp. 382-386, 1986.

[54] J. L. Fleiss, Statistical Methods for Rates and Proportions, John Wiley \& Sons, New York, NY, USA, 2nd edition, 1981.

[55] D. F. Polit and C. T. Beck, Essentials of Nursing Research: Methods, Appraisal, and Utilization, p. 514, Lippincott Williams \& Wilkins, Philadelphia, PA, USA, 2006. 


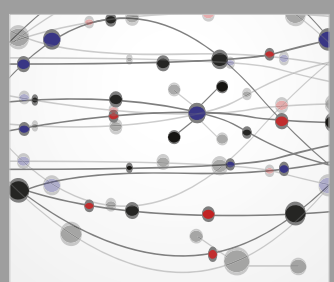

The Scientific World Journal
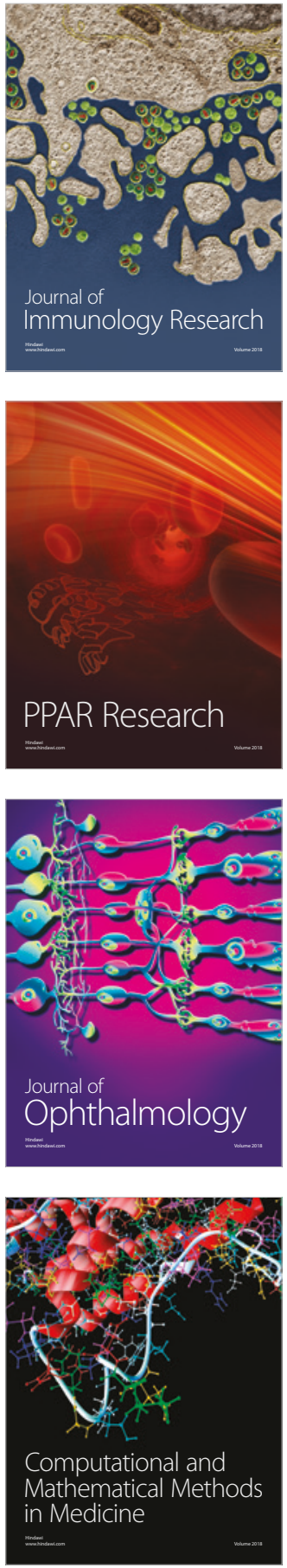

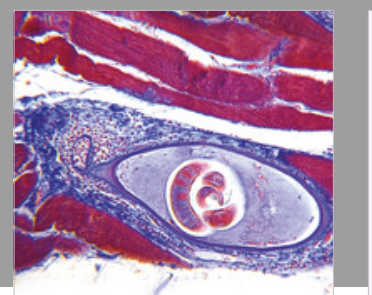

Gastroenterology Research and Practice

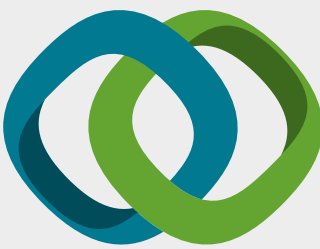

\section{Hindawi}

Submit your manuscripts at

www.hindawi.com
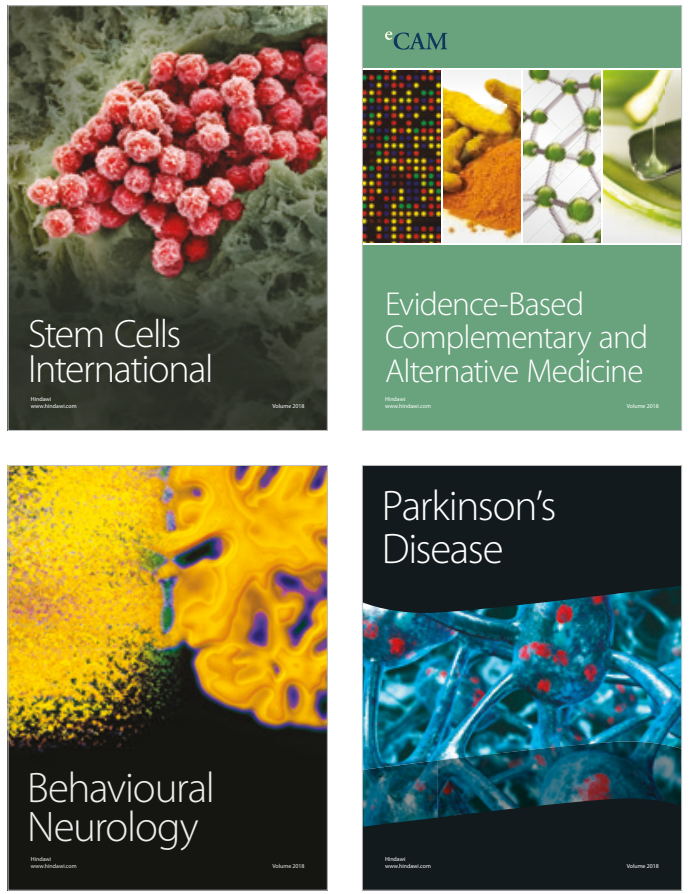

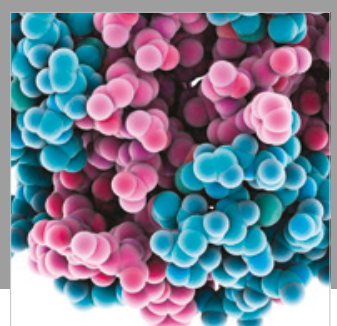

ournal of

Diabetes Research

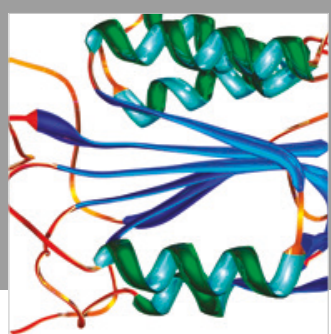

Disease Markers
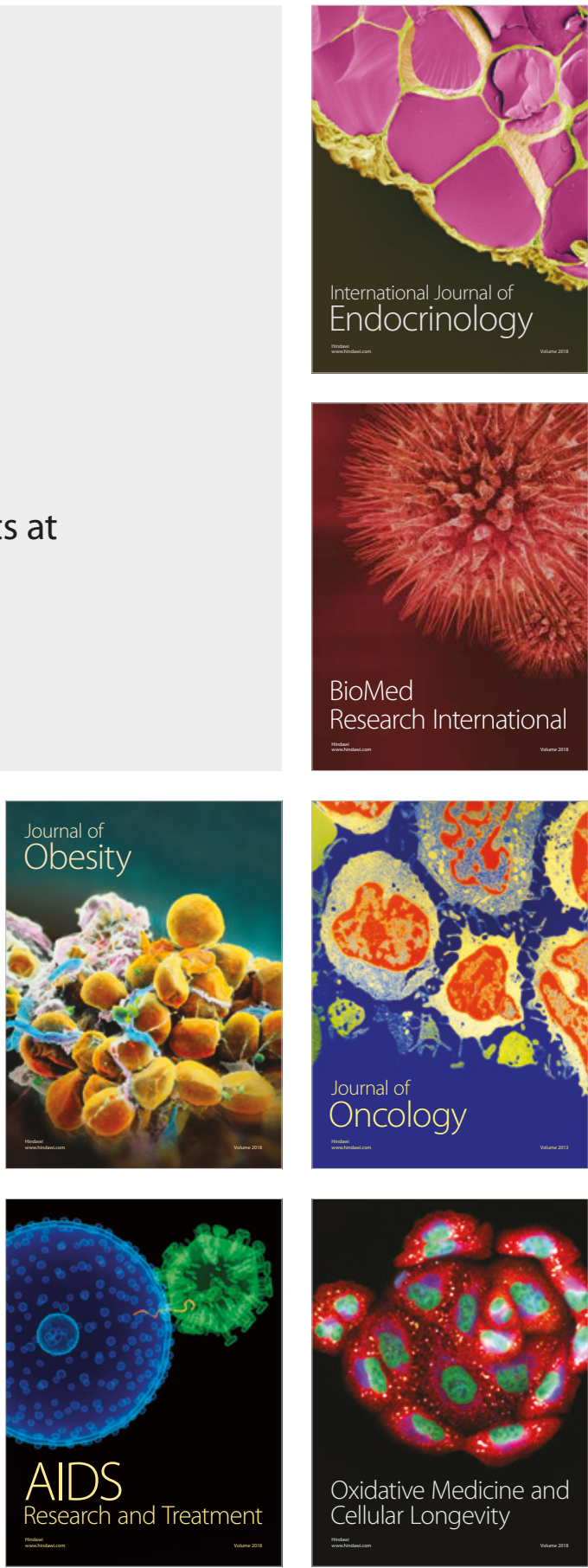